\title{
Discontinuation of tyrosine kinase inhibitors in chronic myeloid leukemia patients - worldwide battlefield
}

\author{
Minireview
}

P. DVORAK ${ }^{1,2}$, D. LYSAK 3 , S. VOKURKA 3

${ }^{1}$ Institute of Biology, Faculty of Medicine in Pilsen, Charles University in Prague, Karlovarska 48, 30166 Pilsen, Czech Republic; ${ }^{2}$ Institute of Medical Genetics, University Hospital Pilsen, Dr. Edvarda Benese 13, 30599 Pilsen, Czech Republic; ${ }^{3}$ Department of Haematology and Oncology, University Hospital Pilsen, alej Svobody 80, 30460 Pilsen, Czech Republic

*Correspondence: Pavel.Dvorak@lfp.cuni.cz

Received May 6, 2014 / Accepted July 14, 2014

\begin{abstract}
Advances in understanding the pathogenesis of chronic myeloid leukemia (CML) and implementation of the therapy with tyrosine kinase inhibitors (TKI) could be considered as a prototype of successful fight against cancer. However, for an optimally responding patient it is recommended to follow the TKI therapy indefinitely. The question about the possibility of safe TKI treatment discontinuation in certain clinical situations was raised and is currently under close investigation worldwide. Currently, imatinib discontinuation trails have shown that about $60 \%$ of eligible patients experienced molecular recurrence within 6 months of treatment discontinuation, while the remaining $40 \%$ remained in defined deep molecular response throughout the duration of mostly two years follow-up. Interestingly, retreatment with the same TKI or another TKI was successful in the vast majority of patients demonstrating molecular recurrence of the disease. These findings support the concept of safe TKI treatment discontinuation and its usefulness for a specific subset of CML patients. However, recent data are not sufficient for TKI discontinuation attempts outside clinical trials yet. Because of the high risk of potentially problematic molecular recurrences of the pathological clones, the key question is to find the right predictive marker of TKI discontinuation success, however it stays unsolved yet. This minireview brings a concise summary of this hot topic with a realistic view from clinical routine.
\end{abstract}

Key words: tyrosine kinase inhibitors, imatinib, discontinuation, chronic myeloid leukemia

Chronic myeloid leukemia (CML) is a clonal hematopoietic stem cell disorder consequent to the origin of the $B C R-A B L 1$ fusion gene, which is predominantly generated by a $\mathrm{t}(9 ; 22)(\mathrm{q} 34 ; \mathrm{q} 11)$ reciprocal translocation. The product of the oncogene, bcr-abll fusion protein, has a constitutive tyrosine kinase activity and is believed to play a crucial role in the development of CML. Biological therapy with tyrosine kinase inhibitors (TKIs) specifically disables this activity and effectively clears malignant clone in the vast majority of CML patients. Imatinib mesylate, first-generation TKI, has become the standard first-line treatment of chronic phase CML during the last decade. Second-generation TKIs (dasatinib, nilotinib) were shortly developed to overcome the resistance or intolerance to imatinib. Clinical trials with next generation TKIs (bosutinib, ponatinib) are in progress.
Several studies of imatinib as first-line therapy have published comparable long-term results. The estimated event-free survival at 6 years was $83 \%$, and the estimated rate of freedom from progression to accelerated phase and blast crisis was $93 \%$ in the IRIS study [1]. Practice guidelines issued by the European LeukemiaNet (ELN) and the National Comprehensive Cancer Network (NCCN) both recommend that a patient who is responding optimally to TKI treatment continues indefinitely at the standard recommended dose $[2,3]$. However, some situations in clinical routine raised the question about the possibility of safe TKI treatment discontinuation. These situations involve issues like chronic toxicity, patient compliance, pregnancy or patient's wish. Furthermore, financial issues of TKI treatment are being considered not only in economies with limited financial 
sources (approximately 5,000 new cases are diagnosed with CML in Europe each year).

Rare cases with long-term stable minimal residual disease or complete molecular response after cessation of interferon alpha therapy had been reported and they laid the theoretical basis for the TKI discontinuation attempts [4]. Soon after 2000, when imatinib treatment came into clinical routine, case reports of patients who stopped the treatment were reported. Few years later, first clinical studies focusing on imatinib dose modification or discontinuation started to be published [5-7].

Response to TKI treatment. At present, the most important prognostic factor for CML patients is the response to TKI treatment. The response is measured based on cytogenetic (Gbanding, FISH) and molecular genetic (qRT-PCR) methods. The definition of optimal response has evolved during the last few years. At present, an optimal response to any TKI as first-line treatment is defined by at least partial cytogenetic response (PCyR; $1-35 \% \mathrm{Ph}+$ metaphases) at 3 months, at least complete cytogenetic response (CCyR; No $\mathrm{Ph}+$ metaphases) at 6 months and at least major molecular response (MMR; $B C R$ $A B L 1: A B L 1 \leq 0.1 \%$ on the International Scale) at 12 months [2]. Molecular response (MR) is mostly assessed according to the International Scale (IS) as the ratio of $B C R-A B L 1$ transcripts to $A B L 1$ transcripts and it is reported as $B C R-A B L 1$ $\%$ on a $\log$ scale, where $10 \%, 1 \%, 0.1 \%, 0.01 \%, 0.0032 \%$, and $0.001 \%$ correspond to a decrease of $1,2,3,4,4.5$, and 5 logs, respectively, below the standard baseline that was used in the IRIS study [2]. A BCR-ABL1 expression of $0.1 \%\left(\mathrm{MR}^{3.0}\right)$ corresponds to major molecular response (MMR). The proportion of patients who achieved MMR after 12 months of $400 \mathrm{mg}$ imatinib daily (standard dose) ranged from $18 \%$ to $58 \%$ in the published studies $[2,3]$. A high number of commercially available kits for molecular genetic analyses from different manufacturers for different platforms are available on the market. It is the responsibility of each laboratory to choose some of the kits to fit with the recommended criteria and obtain results, which are comparable with other laboratories.

Success rate for TKI discontinuation. The nonrandomized multicentre Stop Imatinib (STIM) study was one of the largest prospective studies of TKI treatment discontinuation organized to date [8]. Imatinib treatment was discontinued in patients who maintained $\mathrm{MR}^{5.0}$ (defined as undetectable $B C R-A B L 1$ with assay sensitivity of $\geq 5 \log$ below IS-standardized baseline) for at least 2 years. Of 100 patients enrolled, 61 patients had molecular recurrence after a median of 30 months of follow-up (58 patients within 7 months) and 39 patients maintained $\mathrm{MR}^{5.0}$ after a median of 22 months of follow-up. The overall probability of maintaining $\mathrm{MR}^{5.0}$ at 12 months and 24 months was $43 \%$ and $39 \%$, respectively $[8,9]$. Molecular recurrence was defined as BCR-ABL1 $\geq 0.001 \%$ IS in the STIM study.

The results of another prospective study, TWISTER, were published in 2013 [10]. Imatinib treatment was stopped in 40 patients who maintained $\mathrm{MR}^{4.5}$ for at least 2 years. With a median of 42 months of follow-up post-treatment cessation, 18 patients (45\%) had not demonstrated molecular relapse (defined as loss of MMR or two consecutive occurrences of detectable $B C R-A B L 1$ ), and the Kaplan-Meier estimate of rate of treatment-free remission at 2 years was $47.1 \%[9,10]$. Molecular relapse was defined as loss of MMR or two consecutive occurrences of detectable BCR-ABL1 in TWISTER study.

In the HOVON 51 study, patients received escalating doses of imatinib in combination with escalating doses of cytarabine according to the study protocol. Thirty-three patients from the HOVON 51 study with an $\mathrm{MR}^{4.5}$ for at least 2 years who were still on imatinib treatment were randomized between continuation of imatinib ( $\operatorname{arm~A,~} \mathrm{n}=18$ ) or discontinuation of imatinib ( $\operatorname{arm} B, n=15)$ [11]. After a median follow up of 36 months since randomization, 3 patients (17\%) in arm A and 10 patients (67\%) in arm B had a molecular relapse. All 3 relapsing patients in arm A had also stopped imatinib after randomization. All but one relapsing patient relapsed within 7 months after discontinuation of imatinib. The molecular relapse rate at 12 and 24 months after randomization was $0 \%$ and $6 \%$ (arm A) and $53 \%$ and $67 \%$ (arm B) respectively [11].

Takahashi et al. [12] conducted a nationwide retrospective survey of the clinical outcomes of CML patients after discontinuation of imatinib in Japan. Among 3,242 imatinibtreated CML patients, they identified 50 (1.5\%) who had discontinued imatinib for at least six months; of these they were able to analyze 43 in full. A complete molecular response (CMR) was defined as detection of no $B C R-A B L 1$ transcript in a real-time quantitative-polymerase chain reaction (RQPCR) assay $(n=24)$, nested reverse transcriptase-polymerase chain reaction (RT-PCR) assay $(\mathrm{n}=14)$, or a highly sensitive transcription-mediated amplification (TMA) method $(n=5)$. These PCR methods could detect at least a 4-log reduction in the $B C R-A B L 1$ transcript (international scale $<0.01 \%$ ). The reasons for which imatinib was discontinued were adverse events $(n=18)$, patient's request due to cost $(n=14)$, patient's desire to become pregnant $(n=3)$, and long undetectable residual disease $(n=8)$. The median duration of CMR before cessation was 27.4 months (range 0.9-79.6 months). The median period of cessation was 22.4 months (range 6.2-97.9 months). Molecular recurrence was detected in 19 patients (44\%). The relapse free survival (RFS) rate at five years was estimated to be $47 \%$ while median RFS was determined to be 41 months using the Kaplan-Meier method [12].

The higher rates of deep molecular responses achievable with nilotinib and dasatinib suggest that use of second-generation TKIs might broaden the pool of candidates eligible for treatment discontinuation. To date, only very limited clinical data on discontinuation of nilotinib or dasatinib therapy are available.

Summary of qualification criteria for TKI discontinuation. Above mentioned prospective trails had differed in the qualification criteria for TKI discontinuation and there has not been published any consensus yet. Length of TKI treatment duration, level of response, and duration of response were the 
main criteria used $[8,10,11]$. At least 3 years of TKI treatment duration was demanded in the STIM and TWISTER study. At least $\mathrm{MR}^{4.5}$ for at least 2 years were the minimal required level of response and its duration $[8,10,11]$. The ongoing EURO-SKI study (NCT01596114) has these main inclusion criteria: CML in chronic phase, duration of TKI treatment before enrolment at least 3 years, at least $\mathrm{MR}^{4.0}$ for at least one year (personal communication).

Predictive factors for TKI discontinuation success. Statistically significant predictive factors differed between published studies as well. A trend for a shorter time to $B C R-A B L 1$ negativity in nonrelapsing patients versus relapsing patients was found in the pilot study conducted by Rousselot et al. [6]. The absence of relapse was also not significantly associated with the length of interferon alpha (IFN- $\alpha$ ) exposure prior to imatinib in this study [6]. Highest Sokal score group and female sex were predictive of worsened prognosis, whereas a long duration of imatinib was predictive of improved prognosis in the STIM study conducted by Mahon et al. [8]. Significant interaction with relapse risk was observed only for duration of IFN- $\alpha$ treatment and time to achieve undetectable minimal residual disease after switching from IFN- $\alpha$ to imatinib treatment in the TWISTER study performed by Ross et al. [10]. Three (75\%) of 4 Sokal high-risk patients relapsed vs $45 \%$ of low-risk patients (log-rank $\mathrm{P}>.05)$ in this study [10]. The retrospective study of Takahashi et al. [12] found that based on multivariate regression analysis, imatinib dose intensity and prior IFN- $\alpha$ administration were independently predictive of molecular recurrence within 12 months. The depth of the molecular response should be a factor influencing long-term sustained complete molecular response after discontinuation of imatinib according to this study [12]. Interestingly, duration of prior IFN- $\alpha$ treatment was the most frequently found predictive factor.

Retreatment after relapse. Importantly, in patients who demonstrated molecular recurrence after TKI discontinuation, retreatment with the same TKI or another TKI resulted in high rates of response, mostly as deep as that observed during initial TKI treatment [6-12]. Loss of MMR was recently recommended as a practical and safe criterion for restarting therapy by Rousselot et al. [13]. It is too early to draw any conclusions about survival of continuing versus discontinuing patients.

Limitations of current data. Clinical data on TKI discontinuation obtained from published studies are difficult to compare. The cohorts varied among the clinical studies in many criteria such as how long they had been on TKI therapy, what level of response they had achieved, how long that response had been maintained before discontinuation, and how molecular recurrence was defined. Milder criteria for TKI discontinuation might expand the low pool of candidates, but their heterogeneity may lead to higher rates of relapse and hamper the ability to define the right candidate characteristics. Another limitation of the published studies is the duration of follow-ups, which could not be sufficient to draw relevant conclusions [14]. Moreover, each of the published studies suggested different predictive factors of TKI discontinuation success. Current data about TKI discontinuation don't allow us to predict any long-term consequences of TKI discontinuation and retreatment on the clinical course of CML as well.

Models of operational cure of CML Several models for the achievement of a prolonged drug-free remission in CML are discussed in the literature $[9,14,15]$. The models are not mutually exclusive, and different pathways to drug-free remission might apply in different patients. In the Model of stem cell depletion, there is a rapid initial drop in the level of $B C R$ $A B L 1$, which is thought to reflect the clearance of mature CML progeny that are sensitive to TKI. There follows a second phase with a shallow gradient, which is thought to reflect the gradual depletion of the less-sensitive CML stem cells. The slow but progressive depletion of CML progenitors during TKI treatment may be explained by the apoptosis that is dependent on cell cycling $[14,15]$. In the Model of immunological control, a reduction in the level of minimal residual disease by TKI therapy is sufficient to overcome T-cell anergy and enables the emergence of an autologous immunological response that suppresses, but may not eradicate, the whole CML clone. The risk of relapse on TKI cessation is dependent on the functional immune response and the intrinsic immunogenicity of the CML cells $[14,15]$.

Future directions. Many of the above-mentioned limitations should be solved by numerous ongoing clinical trials conducted or planned worldwide. In Europe these are STIM 2 (imatinib), STOP 2GTKI (nilotinib, dasatinib), EURO-SKI (all TKI's), and German CML Study V (nilotinib), in the United States study organized by the University of Michigan Cancer Center (imatinib) and ENESTgoal (nilotinib), in Japan Dasatinib Stop Trail (dasatinib), in Korea study organized by the Seoul St. Mary's Hospital (imatinib). Other trails are organized by the pharmaceutical companies - Bristol-Myers Squibb (DASFREE, dasatinib), Novartis Pharmaceuticals (ENESTop and ENESTfreedom, both nilotinib). Furthermore, French investigators from around the STop IMatinib (STIM) trial have started to explore the feasibility of second attempt to discontinue imatinib in patients with second sustained CMR who experienced molecular recurrence after first attempt of imatinib discontinuation [16]. Their preliminary data suggest that such a second attempt could be possible and could be beneficial for a small subset of patients [16].

Leukemic stem cells are believed to play a crucial role in leukemic hematopoiesis. Strategies to eradicate both proliferating mature progeny and stem cells will continue to be a focus of much clinical research in the field of CML. Research efforts have focused mainly on combination approaches - TKIs plus other drugs.

Despite the remarkable success of TKIs against bcr-abl1, secondary resistance develops in a significant subset of patients, most often due to point mutations in the BCR-ABL1 tyrosine kinase domain or insensitivity of leukemic stem cells to TKIs [17]. One of the current approaches to overcome this 
resistance is launch of new non-TKI drugs with a different mechanism of action. One of them is the natural alkaloid omacetaxine mepesuccinate that inhibits protein synthesis and induces cell death independently from the presence of bcr-abl1 mutations [18].

\section{Conclusions}

In general, imatinib discontinuation trails have shown that about $60 \%$ of eligible patients experienced molecular recurrence within 6 months of treatment discontinuation, while the remaining $40 \%$ remained in defined deep MR throughout the duration of mostly two years follow-up. It seems certain that achievement of an MMR is not enough to decide on TKI treatment discontinuation and deeper MR is needed. Interestingly, retreatment with the same TKI or another TKI was successful in the vast majority of patients demonstrating molecular recurrence of the disease. These findings support the concept of safe TKI treatment discontinuation and its usefulness for a specific subset of CML patients. Regardless of economic issues, major benefits from TKI treatment discontinuation could have patients with adverse events and pregnant women. However, recent data are not sufficient for TKI discontinuation attempts outside clinical trials yet. Furthermore, the high risk of potentially problematic molecular relapse can be only hardly acceptable for many of the optimally responding patients, who don't experience any significant discomfort connected with the TKI therapy. The key issue is to find the right predictive marker of TKI discontinuation success and it waits for the results of the current research.

Acknowledgements: The authors would like to thank Martin Pesta (Institute of Biology, Faculty of Medicine in Plzen) and Pavel Soucek (Institute of Public Healths in Praha) for their inspiring comments. This work was supported in part by the grants NT 14227 from the Ministry of Health of the Czech Republic and SVV-2014-SVV260 050 from the Faculty of Medicine in Plzen, Charles University in Praha (LF UK Plzen).

\section{References}

[1] HOCHHAUS A, O’BRIEN SG, GUILHOT F, DRUKER BJ, BRANFORD S, et al.. Six-year follow-up of patients receiving imatinib for the first-line treatment of chronic myeloid leukemia. Leukemia 2009; 23: 1054-1061. http://dx.doi. org/10.1038/leu.2009.38

[2] BACCARANI M, DEININGER MW, ROSTIG, HOCHHAUS A, SOVERINI S, et al.. European LeukemiaNet recommendations for the management of chronic myeloid leukemia: 2013. Blood 2013; 122: 872-884. http://dx.doi.org/10.1182/ blood-2013-05-501569

[3] NATIONAL COMPREHENSIVE CANCER NETWORK. NCCN clinical practice guidelines in oncology: chronic myelogenous leukemia, v.4.2013.

[4] KANTARJIAN HM, O'BRIEN S, CORTES JE, SHAN J, GILES FJ, et al.. Complete cytogenetic and molecular responses to interferon-alpha-based therapy for chronic myelogenous leukemia are associated with excellent long-term prognosis. Cancer 2003; 97: 1033-1041. http://dx.doi.org/10.1002/ $\underline{\text { cncr.11223 }}$

[5] ISHIKAWA I, KATO C, HARIGAE H, SUGAWARA T, TOMIYA Y, et al.. Dose modification of imatinib by monitoring the level of BCR-ABL transcript in chronic myelogenous leukemia. Tohoku J Exp Med 2006; 210: 355-363. http:// dx.doi.org/10.1620/tjem. 210.355

[6] ROUSSELOT P, HUGUET F, REA D, LEGROS L, CAYUELA $\mathrm{JM}$, et al.. Imatinib mesylate discontinuation in patients with chronic myelogenous leukemia in complete molecular remission for more than 2 years. Blood 2007; 109: 58-60. http:// dx.doi.org/10.1182/blood-2006-03-011239

[7] GOH HG, KIM YJ, KIM DW, KIM HJ, KIM SH, et al.. Previous best responses can be re-achieved by resumption after imatinib discontinuation in patients with chronic myeloid leukemia: implication for intermittent imatinib therapy. Leuk Lymphoma 2009; 50: 944-951. http:// dx.doi.org/10.1080/10428190902926973

[8] MAHON FX, RÉA D, GUILHOT J, GUILHOT F, HUGUET F, et al.. Discontinuation of imatinib in patients with chronic myeloid leukaemia who have maintained complete molecular remission for at least 2 years: the prospective, multicentre Stop Imatinib (STIM) trial. Lancet Oncol 2010; 11: 1029-1035. http://dx.doi.org/10.1016/S1470-2045(10)70233-3

[9] MAURO MJ. Striving to achieve safe, permanent treatment discontinuation in chronic myeloid leukemia. Leuk Res 2013; 37: 1395-1403. http://dx.doi.org/10.1016/j. leukres.2013.07.029

[10] ROSS DM, BRANFORD S, SEYMOUR JF, SCHWARER AP, ARTHUR C, et al.. Safety and efficacy of imatinib cessation for CML patients with stable undetectable minimal residual disease: results from the TWISTER Study. Blood 2013; 122: 515-522. http://dx.doi.org/10.1182/blood-2013 $-02-483750$

[11] THIELEN N, VAN DER HOLT B, CORNELISSEN JJ, VERHOEF GE, GUSSINKLO T, et al.. Imatinib discontinuation in chronic phase myeloid leukaemia patients in sustained complete molecular response: a randomised trial of the DutchBelgian Cooperative Trial for Haemato-Oncology (HOVON). Eur J Cancer 2013; 49: 3242-3246. http://dx.doi.org/10.1016/j. ejca.2013.06.018

[12] TAKAHASHI N, KYO T, MAEDA Y, SUGIHARA T, USUKI $\mathrm{K}$, et al.. Discontinuation of imatinib in Japanese patients with chronic myeloid leukemia. Haematologica 2012; 97: 903-906. http://dx.doi.org/10.3324/haematol.2011.056853

[13] ROUSSELOT P, CHARBONNIER A, CONY-MAKHOUL P, AGAPE P, NICOLINI FE, et al.. Loss of major molecular response as a trigger for restarting tyrosine kinase inhibitor therapy in patients with chronic-phase chronic myelogenous leukemia who have stopped imatinib after durable undetectable disease. J Clin Oncol 2014; 32: 424-430. http://dx.doi. org/10.1200/JCO.2012.48.5797

[14] TANG M, FOO J, GÖNEN M, GUILHOT J, MAHON FX, MICHOR F. Selection pressure exerted by imatinib therapy leads to disparate outcomes of imatinib discontinuation 
trials. Haematologica 2012; 97: 1553-1561. http://dx.doi. org/10.3324/haematol.2012.062844

[15] MELO JV, ROSS DM. Minimal residual disease and discontinuation of therapy in chronic myeloid leukemia: can we aim at a cure? Hematology Am Soc Hematol Educ Program 2011; 2011: 136-142.

[16] LEGROS L, ROUSSELOT P, GIRAUDIER S, TULLIEZ M, HUGUET F, et al.. Second attempt to discontinue imatinib in CP-CML patients with a second sustained complete molecular response. Blood 2012; 120: 1959-1960. http://dx.doi. org/10.1182/blood-2012-02-408229
[17] JABBOUR EJ, CORTES JE, KANTARJIAN HM. Resistance to tyrosine kinase inhibition therapy for chronic myelogenous leukemia: a clinical perspective and emerging treatment options. Clin Lymphoma Myeloma Leuk 2013; 13: 515-529. http://dx.doi.org/10.1016/j.clml.2013.03.018

[18] KANTARJIAN HM, O'BRIEN S, CORTES J. Homoharringtonine/omacetaxine mepesuccinate: the long and winding road to food and drug administration approval. Clin Lymphoma Myeloma Leuk 2013; 13: 530-533. http://dx.doi.org/10.1016/j. clml.2013.03.017 